\title{
Axotomy-induced alterations in the red nucleus revealed by monoclonal antibody, Py, following a low thoracic spinal cord lesion in the adult rat
}

\author{
GA Brook ${ }^{1}$, W Nacimiento ${ }^{1}$, A-S Taheri ${ }^{1}$, PL Woodhams ${ }^{2}$ and $\mathrm{J}$ Noth $^{1}$ \\ ${ }^{1}$ Department of Neurology, Aachen University Medical School, Pauwelsstraße 30, 52057-Aachen, Germany; ${ }^{2}$ Norman \\ and Sadie Lee Research Centre, Division of Neurobiology, National Institute for Medical Research, Mill Hill, London \\ NW7 IAA, UK
}

The monoclonal antibody Py was previously developed as a tool for the identification of subpopulations of hippocampal neurons. Here, the differential distribution of Py immunoreactivity in the mid-brain is described showing that Py also serves as a useful marker for other populations of neurons. Medium to strong immunoreactivity was observed in the cell body and dendrites of neurons of the oculomotor nucleus, superior colliculus and substantia gelatinosa reticulata. However, particularly intense Py-immunoreactivity was identified in the magnocellular neurons in the caudal pole of the red nucleus. Unilateral transection of the rubrospinal tract at Th9-10 induced a marked reduction of Py immunoreactivity in the ventrolateral territory of the caudal pole of the axotomised red nucleus. A small but statistically significant reduction of Py-immunoreactivity was first seen at 7 days after surgery and a maximal loss of immunoreactivity (reduced to $66 \%$ of control levels) was observed by 21 days after surgery. Immunoreactivity in the axotomised red nucleus was reduced for the duration of the experiment but at the longer survival times studied ( 3 and 6 months) a small degree of recovery of staining was observed in small-medium diameter atrophic neurons. These results indicate that monoclonal antibody Py, may be a useful novel and sensitive tool for investigating the cell body reaction of particular populations of axotomised CNS neurons following spinal cord injury.

Keywords: red nucleus; axotomy; spinal cord injury

\section{Introduction}

Monoclonal antibody Py, which binds to a $146 \mathrm{kDa}$ glycoprotein, was developed as a marker for the selective identification of sub-populations of neurons of the adult rat hippocampus. ${ }^{1}$ Recently, a differential pattern of Py-immunoreactivity (IR) has been documented in the normal adult rat spinal cord. Intense Pystaining was detected in the cell body and dendrites of large neurons, particularly motoneurons and Clarke's nucleus neurons. ${ }^{2}$ Subsequent studies in the lesioned spinal cord have indicated that Py may also be a useful tool for studying the cell body response of axotomised populations of neurons. Py-IR was rapidly lost in Clarke's nucleus neurons ipsilateral and caudal to a unilateral destruction of the dorsal spinocerebellar tract. $^{2}$ However, it is not certain whether the loss of Py-staining demonstrates a specific aspect of the cell body response to axotomy or if this reflects a more generalised phenomenon during the processes of neuronal degeneration.

In an attempt to resolve this question, possible alterations in Py-IR have been studied in axotomised

Correspondence: GA Brook rubrospinal neurons. The red nucleus is a conspicuous bilateral structure in the mid-brain tegmentum which projects to the contralateral spinal cord via the dorsolateral fasciculus. $^{3,4}$ Axotomised rubrospinal neurons have been reported to undergo atrophic rather than degenerative changes following a midlow thoracic spinal cord injury ${ }^{5-11}$ and the contralateral (non-lesioned) region of the red nucleus may be used as an internal control for side-to-side comparisons.

Here we extend current knowledge concerning PyIR in the adult rat CNS by demonstrating its differential distribution in neurons of the mid-brain and show that axotomised magnocellular neurons of the red nucleus undergo a rapid reduction of immunoreactivity.

\section{Methods}

The investigation was carried out on a total of 23 adult female Sprague-Dawley rats $(200-250 \mathrm{~g})$. Spinal cords and brains were processed for immunohistochemical investigation at 4, 7, 14 and 21 days, 3 and 6 month post operation (po) with three animals in each survival 
time. Three normal (unoperated) and two shamoperated animals ( 3 months survival time) were used for controls.

\section{Surgical procedure}

Animals were deeply anaesthetized by intraperitoneal (ip) injection of chloral hydrate $(350 \mathrm{mg} / \mathrm{kg}$ body weight). The dorsal and lateral surfaces of the spinal cord were exposed by laminectomy at level Th8-9 and a lateral funiculotomy of the left side of the cord was performed. Haemostasis was established and the muscle and skin closed in layers. In sham operated animals laminectomy was followed by incision of the dura without lesioning the spinal cord.

\section{Tissue processing for immunohistochemistry}

At a range of survival times, rats were given terminal anaesthesia (chloral hydrate, $640 \mathrm{mg} / \mathrm{kg}$ ip) and were perfused, via the left ventricle, with $0.9 \%$ saline $(100 \mathrm{ml})$ followed by $4 \%$ paraformaldehyde containing $15 \%(\mathrm{v} / \mathrm{v})$ saturated picric acid in $0.1 \mathrm{M}$ phosphate buffer (PB, pH 7.4). The spinal cord (including the lesion site) and brain were rapidly dissected and postfixed in $4 \%$ paraformaldehyde $\left(24 \mathrm{~h}, 4^{\circ} \mathrm{C}\right)$. Tissue was then cryoprotected in $30 \%$ sucrose (overnight, $4^{\circ} \mathrm{C}$ ) prior to being frozen in isopentane $\left(-80^{\circ} \mathrm{C}\right)$.

\section{Assessment of lesions}

Serial transverse sections of the lesion site were stained with thionin to assess the accuracy and extent of each lesion. Animals were not included in the analysis if the operated lateral fasciculus was partially spared or if the non-operated lateral fasciculus had been damaged. Following reconstruction of the lesion site, the brains of three rats per survival time (with confirmed lateral funiculotomy) were processed further for the immunohistochemical analysis. The results obtained from all these animals are described.

\section{Immunohistochemistry for Py}

For paraformaldehyde fixed brains, serial coronal cryostat sections $(20 \mu \mathrm{m}$ or $8 \mu \mathrm{m}$ thick) were collected individually into $0.1 \mathrm{M} \mathrm{PB}$ in plastic microwells. While cutting, particular care was exercised to ensure that sections were as coronal as possible by assessing the bilateral symmetry of structures in the brain. Following the blocking stage of incubation in $3 \%$ normal goat serum (30 min), sections were incubated overnight in primary antibody (diluted 1:20000 containing $0.1 \%$ Triton $\mathrm{X}-100$ ) at room temperature with intermittent agitation. The next day, sections were washed in $0.1 \mathrm{M} \mathrm{PB}$ and incubated in secondary antibody (biotinylated goat anti-mouse IgM, diluted $1: 1000$ ) followed by the Vector ABC procedure for peroxidase staining and visualisation with 3,3'diaminobenzidine (DAB). Omission of primary anti- body was used for negative controls. The $8 \mu \mathrm{m}$ sections were counterstained with thionin $(0.02 \%)$ prior to coverslipping.

\section{Quantification of lesion induced alterations using densitometric analysis}

The mean optical density of $\mathrm{DAB}$ reaction product in the caudal pole of the lesioned and non-lesioned red nucleus was measured by using a computerized image analysis programme, as described previously. ${ }^{12}$ Values were taken from ten sections per animal and intraanimal right/left side ratios (ie affected/unaffected side) were determined. Analyses of the normal distribution and variance of results were made. If a normal distribution and equal variance was observed between groups, the paired Student $t$ test was applied to assess the statistical significance; if a normal distribution or equal variance was not observed, the Wilcoxon test was applied.

\section{Results}

Distribution of $P y-I R$ in the normal rat mid-brain Py-immunoreactivity displayed a differential pattern of staining in the mid-brain allowing the territory occupied by several nuclei to be distinguished. Regions showing strong Py-IR included the red nucleus, oculomotor nuclei, superficial and intermediate gray layers of the superior colliculus and the interpeduncular nucleus. Moderately intense staining was observed in the substantia nigra pars reticulata and deep mesencephalic nucleus. Some degree of compartmentalisation of the medial geniculate nucleus could be observed since the dorsal territory was Py-negative and the ventral territory weakly Py-positive.

The dense packing of strongly stained neuronal perikarya and dendrites clearly delineated the territory occupied by the caudal (magnocellular) pole of the red nucleus (Figure $1 \mathrm{a}-\mathrm{c}$ ). Densitometric analysis indicated that, in control animals, the caudal pole of the red nucleus displayed bilaterally symmetrical Py-IR (Table 1). The rostral (parvicellular) region of the red nucleus was less well defined since cell bodies and dendrites were less densely packed, and no distinct border could be observed between the dorsal and lateral aspects of the nucleus and the adjacent neuropil (Figure 1d).

Immunoreactivity appeared to be confined to the cytoplasm of neuronal profiles. The nucleus was unstained. In the red nucleus, immunoreactivity was most intense in the cell body and dendrites of the large diameter $(25-50 \mu \mathrm{m})$ magnocellular neurons. As described in other regions of the CNS, ${ }^{1,2}$ when sections were viewed at high magnification the staining appeared filamentous in the proximal portion of large diameter primary dendrites. The neurons in the ventrolateral region of the nucleus appeared to be slightly larger and more intensely stained than those in 
the dorsomedial region (Figure 1e). Thin $(8 \mu \mathrm{m})$ sections, counterstained with thionin, revealed that all the large diameter cells were intensely Py-IR. The medium and small diameter neurons in the red nucleus were also Py-positive, but generally, the staining was much weaker than in larger neurons (Figure 1f). No immunoreactivity could be detected in non-neuronal elements, such as the glia, ependymal cells or endothelial cells (Figure 3c).
$P y-I R$ in the red nucleus after lesion of the rubrospinal tract at low thoracic level

Reconstruction of the lesion site indicated that all low thoracic hemisections resulted in a unilateral destruction of the rubrospinal tract. Occasionally, degeneration also included part of the dorsal funiculus and the contralateral gray matter. The lateral funiculus located on the unoperated side of the spinal cord always remained intact.
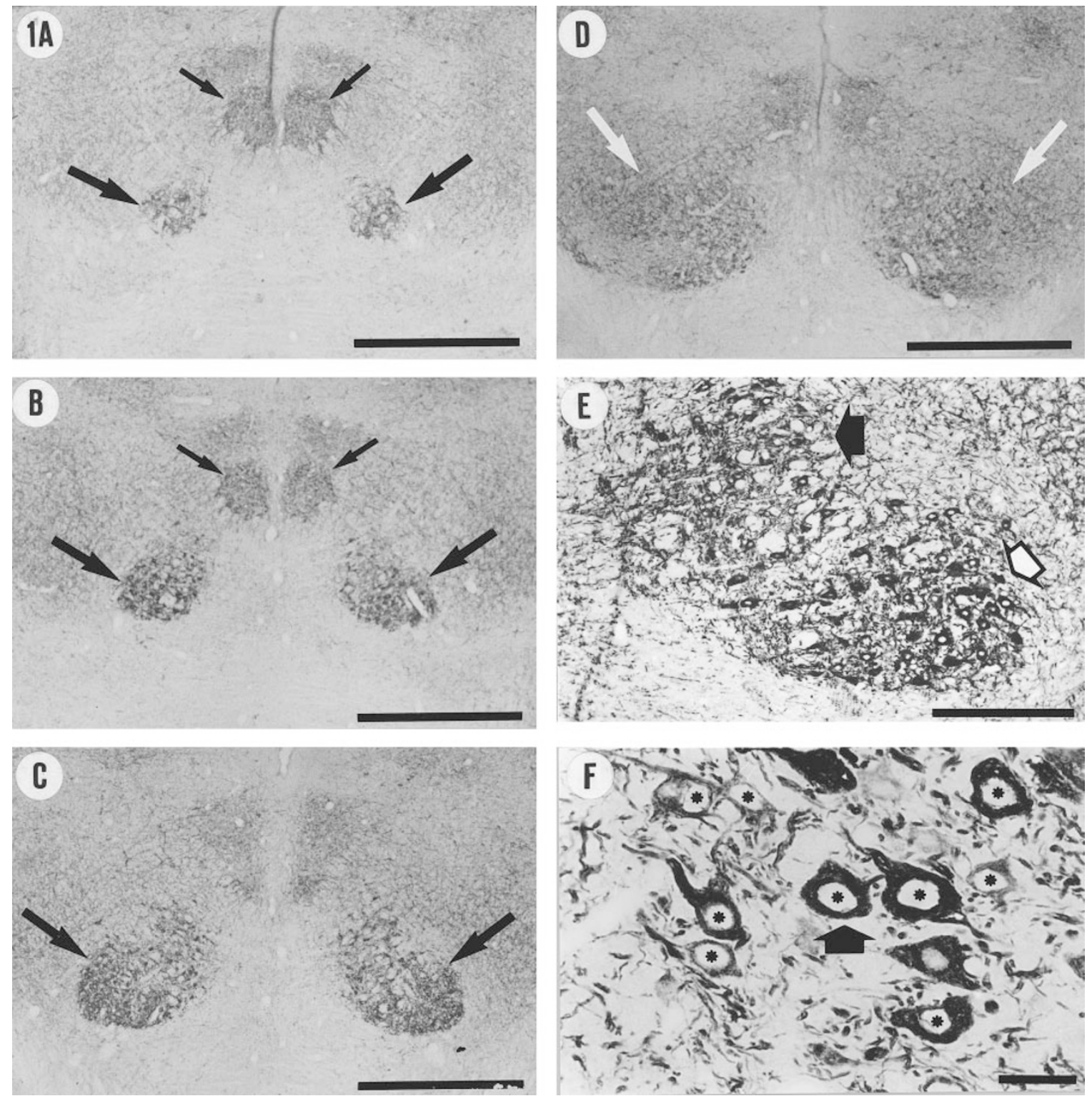

Figure 1 (a-f) Coronal $20 \mu \mathrm{m}$ sections of the mid-brain showing the distribution of Py immunoreactivity in the normal red nucleus. (a) $50 \mu \mathrm{m}$; (b) $200 \mu \mathrm{m}$; (c) $350 \mu \mathrm{m}$; (d) $650 \mu \mathrm{m}$ from caudal pole of the red nucleus. Py immunoreactivity reveals the bilateral symmetry of intensely Py immunoreactive oculomotor nucleus (small arrows) and red nucleus (large arrows). The caudal (magnocellular) region of the red nucleus (large black arrows) is most intensely Py positive. The rostral (parvicellular) region is less intensely stained (white arrows). (e) Large, intensely stained magnocellular neurons concentrated in the ventrolateral region of the red nucleus (open arrow), less intensely stained dorsomedial region (closed arrow). (f) Py immunoreactivity is most intense in the large neurons (arrow), smaller neurons are more weakly stained. Neuronal nuclei (asterisks) are unstained. $8 \mu \mathrm{m}$ thick coronal section. Scale bars a-d: $1 \mathrm{~mm}$, e: $300 \mu \mathrm{m}, \mathrm{f}: 50 \mu \mathrm{m}$ 
Table 1 Side-to-side comparison of Py-IR in the red nucleus following unilateral lesion of the rubrospinal tract

\begin{tabular}{lcccccccc}
\hline & CONT & SHAM & 4 dpo & 7 dpo & 14 dpo & 21 dpo & 3 mpo & 6 mpo \\
\hline $\begin{array}{l}\text { Mean } \\
(\%)\end{array}$ & 100.7 & 101.1 & 100.29 & 88.61 & 79.4 & 66.10 & 80.99 & 73.42 \\
SD & & & & & & & & \\
P value & 3.5 & 6.9 & 7.96 & 9.55 & 8.73 & 15.58 & 7.53 & 11.42 \\
\hline
\end{tabular}

Values are expressed as mean of the percentage of Py-IR observed in the right (axotomised) as compared to the left (nonaxotomised) red nucleus in control (CONT), sham operated (SHAM), and lesioned animals. Survival times indicated in days po (dpo) or months po (mpo). Standard deviations (SD) are also indicated
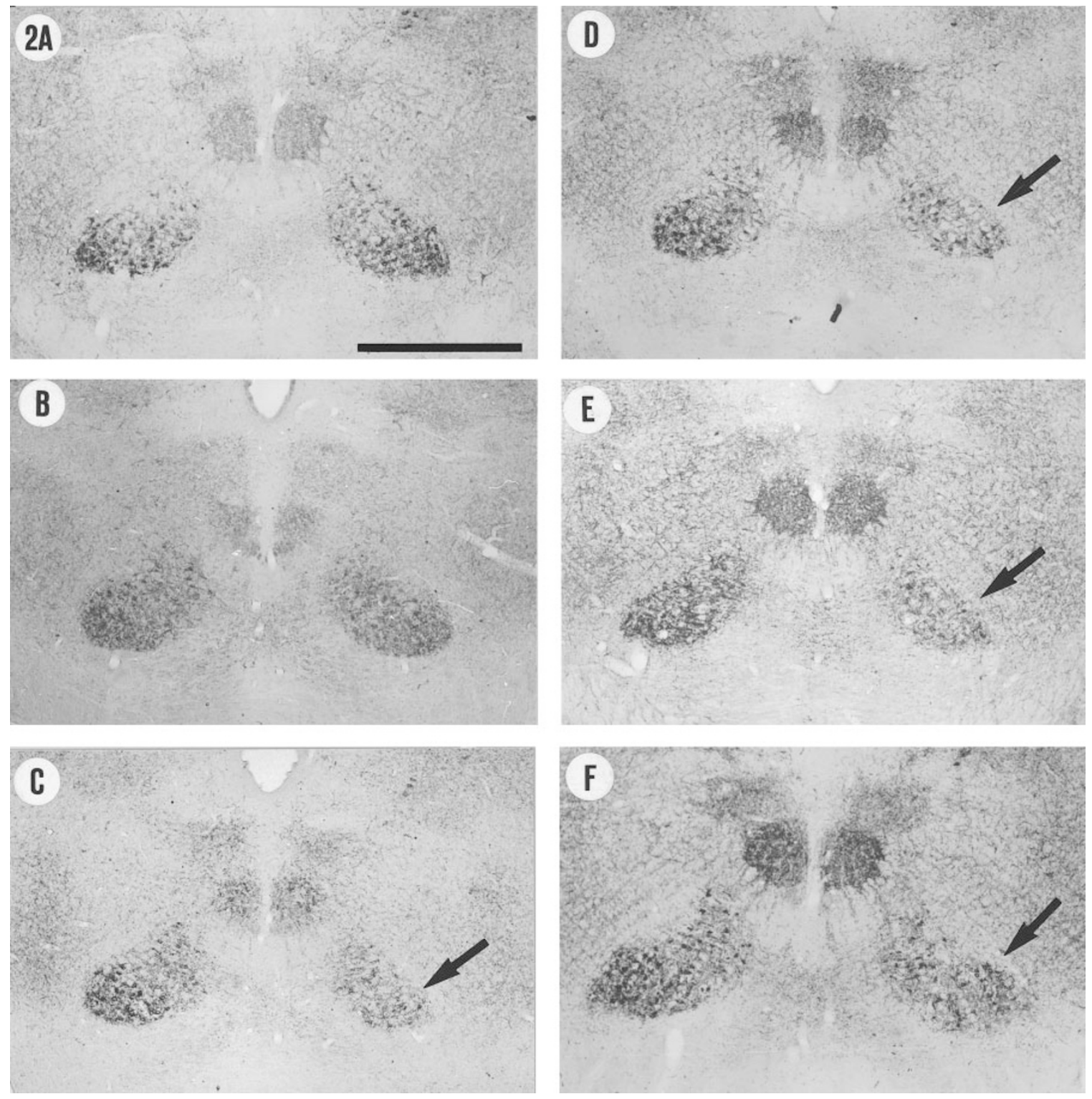

Figure 2 (a-f) Coronal $20 \mu \mathrm{m}$ sections showing axotomy induced reduction of Py immunoreactivity in the caudal (magnocellular) region of the red nucleus. (a) 4 days po; (b) 7 days po; (c) 14 days po; (d) 21 days po; (e) 3 months po; (f) 6 months po. Axotomised red nucleus is always on the right. There was no apparent alteration in the level of Py immunoreactivity for the first 4 days after lesion of the rubrospinal tract. However, a clear reduction of Py staining could be seen from 14 days po (arrows). Scale bar: $1 \mathrm{~mm}$ 
No clear evidence of statistically significant alterations to the intensity or distribution of Py staining could be seen at 4 days po (Figure 2a and Table 1). However, by 7 days po, densitometric analysis revealed a small but statistically significant reduction of Py-IR when compared to the contralateral non-axotomised magnocellular region of the red nucleus (reduced to $88 \%$ of control level, $P<0.001$, Figure $2 \mathrm{~b}$ and Table 1 ). The reduction of Py-IR progressed for the subsequent 2 weeks reaching a maximal loss of immunoreactivity by 21 days po $(66 \%$ of control level, $P<0.001$, Figure $2 \mathrm{c}$, $\mathrm{d}$ and Table 1). The loss of Py-IR was restricted to the caudal pole of the red nucleus and was most striking in the ventrolateral region, where the dense arborization of intensely stained dendrites could no longer be detected. At this survival time weakly stained, medium-large diameter $(25-50 \mu \mathrm{m})$ magnocellular neurons were observed (Figure 3c). Immunoreactivity was never completely lost in the ventrolateral region of the axotomised red nucleus. The perikarya and dendrites of small, medium and occasional large diameter neurons could be observed at all survival times. The neurons in the dorso-medial aspect of the red nucleus appeared largely unaffected by transection of the rubrospinal tract at low thoracic levels.

The lesion induced reduction of Py-IR was persistent throughout the duration of the experiment (Figure 2e, f). However, at the longest survival times ( 3 and 6 months po) there was a small recovery of immunoreactivity towards control levels (Table 1). At these survival times, an unusually strong signal could be detected in many small-medium diameter neurons in the ventrolateral region of the red nucleus (compare Figure $3 \mathrm{a}$ and $\mathrm{b}$ ).

The post-lesional changes of Py-IR were always confined to the red nucleus contralateral to the injury. No alteration of Py-IR could be observed after sham operations.
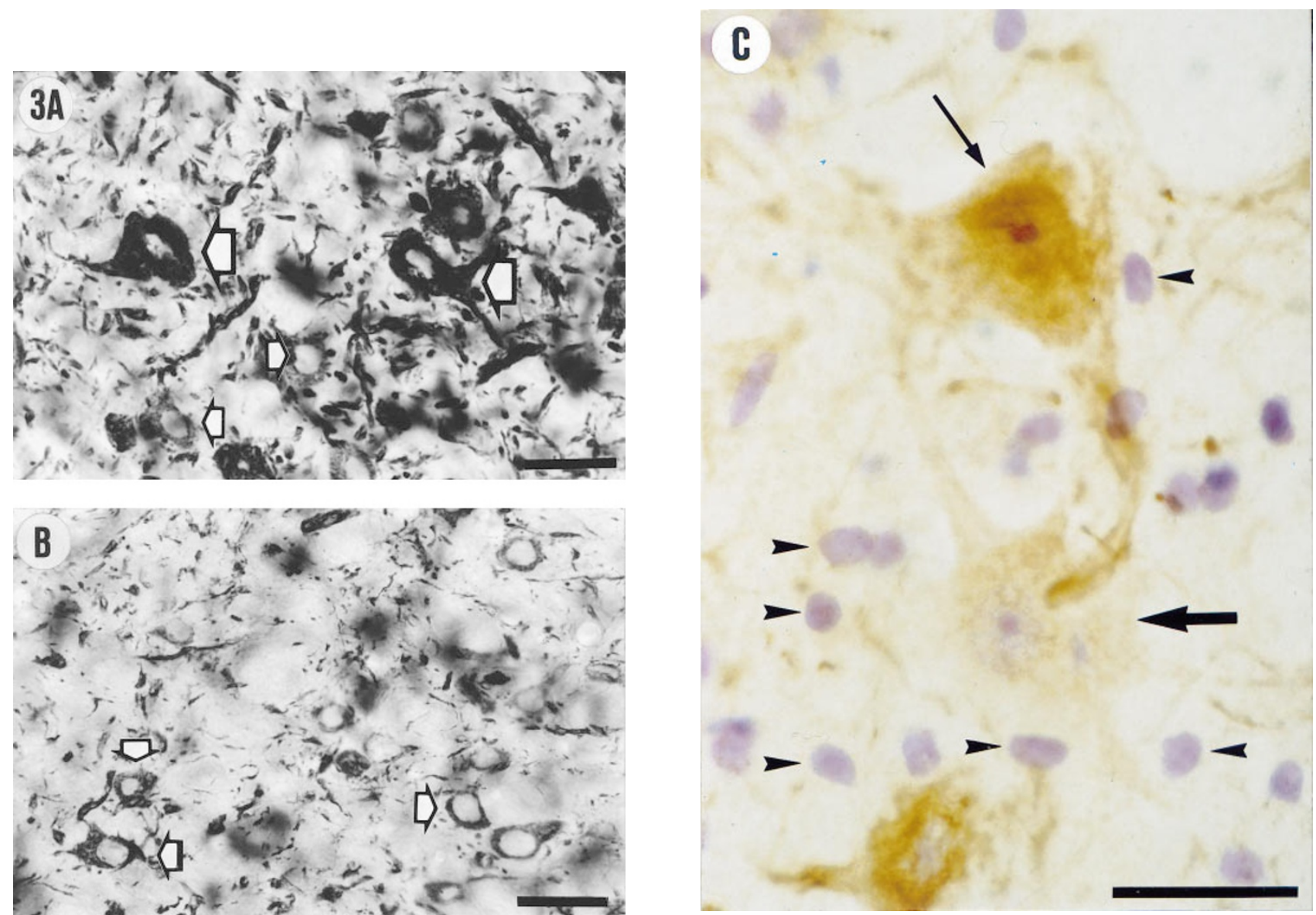

Figure 3 Py immunoreactivity in the ventrolateral region of the non-axotomised (a) and axotomised (b) red nucleus 6 months after lesion of the rubrospinal tract. Small diameter neurons (open arrows in b) display an uncharacteristically strong Pyimmunoreactivity and may represent atrophic rubrospinal neurons. Compare intensity of staining of small neurons in b with that of large neurons (large arrows) and small neurons (small arrows) in (a). (c) Marked reduction of Py immunoreactivity in cell body of a large, magnocellular neuron (large arrow) at 3 weeks po. Not all large neurons in the region are affected, note the strong Py-staining of the adjacent neuronal cell body (small arrow). Thionin stained nuclei of glial cells (arrowheads) are not associated with Py immunoreactivity. $8 \mu \mathrm{m}$ thick section. Scale bars a, b: $50 \mu \mathrm{m}$, c: $25 \mu \mathrm{m}$ 


\section{Discussion}

Axotomy of intrinsic CNS neurons by spinal cord injury induces a variety of alterations which can be identified by morphological, biochemical and molecular techniques. These neuronal changes are collectively termed the 'cell body reaction' or 'axon reaction', the timing and severity of which depends on a number of factors eg developmental stage of the animal, type of lesion, distance between the cell body and the site of injury and the degree of collateralization proximal to the lesion. ${ }^{6,13-16}$ A component of the cell body response to axotomy involves alterations to the cytoskeleton. The functional significance of cytoskeletal modification and the mechanism(s) controlling such changes remain uncertain. ${ }^{15,17}$ The present data describe a novel component of the cellular alterations in axotomised CNS neurons using the recently developed monoclonal antibody, Py, which binds to a $146 \mathrm{kDa}$ cytoplasmic protein.

The magnocellular neurons of the red nucleus are a popular cell group for investigating axotomy induced alterations because they project to the spinal cord in a compact, readily accessible tract in the lateral fasciculus and their soma are confined to a relatively small area in the midbrain., ${ }^{3,48}$ Differential responses of axotomized magnocellular neurons of the red nucleus have been documented following spinal cord injury. These differential responses are dependent on the distance between the lesion site and the cell body. Traumatic lesion to the rubrospinal tract at cervical level induces rapid and intense chromatolytic alterations of axotomized neurons followed by severe cell body, nuclear and nucleolar atrophy, reduced RNA levels and the death of a relatively small (but variable) number of cells. ${ }^{6,19,20}$ Despite the apparently regressive nature of these changes, lesion of the rubrospinal tract at cervical levels has also been shown to induce a transient expression of the early immediate gene c-Jun and mRNA for certain regeneration-associated molecules. $^{21-23}$

In contrast to the intense alterations induced by lesions at cervical levels, injury to the rubrospinal tract at mid to low thoracic levels fails to induce c-Jun and mRNA for regeneration associated genes, and the axotomized magnocellular neurons undergo mild, reversible chromatolysis, atrophy and the retraction of distal dendritic processes. $5,6,9,10,21-24$ This variation in cell body response, according to the rostro-caudal level of the lesion correlates with the observation that severed rubrospinal axons grow into a peripheral nerve graft only when the graft is inserted at a mid-cervical level and not when it is inserted at mid to low thoracic levels. ${ }^{17,25}$

The relatively mild response of the lumbar projecting rubrospinal neurons to a mid-thoracic spinal cord injury makes this an ideal model for assessing the possible usefulness of the monoclonal antibody Py as a tool for studying aspects of the cell body response of (some) lesioned CNS systems.
Previous investigations have revealed that the Py antigen is located in sub-populations of neurons in the hippocampus, cerebral cortex and cerebellum ${ }^{1}$ in the brain. More recently the distribution of Py-IR has been documented in the spinal cord, where it also shows a differential pattern of staining, being most abundant in cell body and dendrites of large diameter neurons in the Clarke's nucleus and also in $\alpha$ motoneurons. Light and electron microscopic analyses indicated that the antigen recognised by Py was associated with the cytoskeleton, ${ }^{2}$ but further biochemical analyses are being undertaken to confirm this.

Subsequent studies in the lesioned spinal cord have shown that the Py antigen is labile, antigenicity being rapidly lost in axotomised Clarke's nucleus neurons. ${ }^{2}$ The loss of Py-IR in these neurons substantially preceded the ensuing cell death. However, it was not certain whether the loss of Py-staining demonstrated a novel aspect of the cell body response to axotomy or if this reflected an early stage in the processes of neuronal degeneration.

The data presented here strongly suggest that the lesion-induced reduction of Py-IR is a novel component of the cell body response of axotomised rubrospinal neurons. It is interesting that a slight reduction of Py-IR was first detected at the early post operative survival time of 7 days po. Tetzlaff and colleagues have observed a precipitous reduction of actin and total tubulin mRNA which occurs between 7 and 14 days po in axotomised rubrospinal neurons following lesions at cervical levels. ${ }^{21}$ The reduction of Py-IR was restricted to the ventrolateral portion of the red nucleus. This observation is consistent with the topographical organisation of the red nucleus, the dorsomedial and ventrolateral components projecting to cervical/upper thoracic and lumbar/lower thoracic regions of the spinal cord respectively. ${ }^{4,11,26,27}$

It is possible that atrophy of axotomised rubrospinal neurons contributes to the reduction of Py-IR. Recent studies have indicated that 4 weeks after rubrospinal tractotomy, lumbar projecting neurons display atrophic alterations combined with reduction of total dendritic length. ${ }^{28}$ In the present study, weakly Py-IR magnocellular neurons could be identified 2-3 weeks after axotomy. Therefore, it is likely that atrophic alterations and loss of Py-IR from the cell body both contribute to the post-lesional dynamics of Py-IR.

The reduction of Py-IR was persistent throughout the duration of the experiment (although a small degree of recovery of immunoreactivity was observed at 3 and 6 months po). The long term reduction of PyIR may be related to the fact that axotomised rubrospinal axons do not regenerate. At the longest survival time studied, numerous small-medium diameter, strongly Py-IR neurons could be observed in the ventrolateral region of the axotomised red nucleus. The intensity of Py-IR in these neurons was greater than that seen in neurons of a similar diameter in the 
non operated red nucleus. This observation may reflect the beginning of a phase of recovery of Py-IR in the atrophic lumbar projecting rubrospinal neurons. A similar partial recovery in the level of intracellular acetylcholinesterase has also been reported to occur in axotomised rubrospinal neurons at extended survival times. ${ }^{29}$ Preliminary studies have indicated that spinal cord $\alpha$-motoneurons also show a rapid reduction of Py-IR following axotomy (Brook et al., unpublished observations). Work is in progress to determine if the loss of immunoreactivity is reversed by the reestablishment of appropriate synaptic connectivity in the regenerating peripheral nervous system.

The present description extends our current knowledge concerning Py-IR in the adult rat CNS by demonstrating its distribution in the mid-brain. The reason for differential staining of particular subpopulations of neurons in the CNS remains unknown. The observation that the large magnocellular neurons were intensely Py-IR and medium to small neurons were more weakly stained is consistent with previous observations. ${ }^{1,2}$ The filamentous appearance of Py staining in the large primary dendrites of magnocellular rubrospinal neurons also supports similar observations made in the pyramidal neurons, Clarke's nucleus neurons and $\alpha$-motoneurons. ${ }^{1,2}$

The mechanism(s) underlying the reduction of PyIR are at present unknown. It is possible that reduced expression of mRNA for the Py antigen or altered post-translational modification (eg increased or decreased phosphorylation) may play a role in the loss of Py-IR. Examples of such post-lesional changes involving cytoskeletal proteins have been observed in other axotomised intrinsic and extrinsic neurons of the CNS. $^{21,30-34}$

\section{Conclusion}

Although the functional role of the antigen recognised by Py remains unknown, the differential distribution and apparent sensitivity to axotomy suggests that Py may be a useful novel marker for studying the mechanisms involved in cellular alterations of axotomised neurons following spinal cord injury.

\section{Acknowledgements}

Our studies were supported by grants from the DFG (No. 102/2-2), BMBF (Teileprojekt 9) and the Alfred Krupp von Bohlen und Halbach Foundation. The authors wish to thank Ingrid Allekotte for technical assistance and Ullrich Görres for the excellent photographic work. This work forms part of the doctoral thesis of A-S Taheri (D82, Diss RWTH Aachen).

\section{References}

1 Woodhams PL et al. A monoclonal antibody, Py, distinguishes different classes of hippocampal neurons. Journal of Neuroscience 1989; 9: $2170-2181$.
2 Brook GA et al. Differential distribution of immunoreactivity in the adult rat spinal cord revealed by the monoclonal antibody, Py: a light and electron microscopic study. Experimental Neurology (1997) In press.

3 Brown LT. Rubrospinal projections in the rat. Journal of Comparative Neurology 1974; 154: 169-188.

4 Antal $\mathrm{M}$ et al. The termination pattern and postsynaptic targets of rubrospinal fibers in the rat spinal cord: a light and electron microscopic study. Journal of Comparative Neurology 1992; 325: $22-37$.

5 Prendergast J, Stelzner DJ. Changes in the magnocellular portion of the red nucleus following thoracic hemisection in the neonatal and adult rat. Journal of Comparative Neurology 1976; 166: 163 171.

6 Egan DA, Flumerfelt BA, Gwyn DG. Axon reaction in the red nucleus of the rat. Perikaryal volume changes and the time course of chromatolysis following cervical and thoracic lesions. Acta Neuropathologica Berlin 1977; 37: 13 - 19.

7 Barron KD et al. Cytological and cytochemical (RNA) studies on rubral neurons after unilateral rubrospinal tractotomy: the impact of GM1 ganglioside administration. Journal of Neuroscience Research 1989; 22: 331 - 337.

8 Barron KD et al. Perineuronal glial responses after axotomy of central and peripheral axons. A comparison. Brain Research 1990; 523: 219-229.

9 McBride RL et al. Prelabeled red nucleus and sensorimotor cortex neurons of the rat survive 10 and 20 weeks after spinal cord transection. Journal of Neuropathology and Experimental Neurology 1989; 48: $568-576$.

$10 \mathrm{McBride} \mathrm{RL}$ et al. Retrograde transport of fluoro-gold in corticospinal and rubrospinal neurons 10 and 20 weeks after T9 spinal cord transection. Experimental Neurology 1990; 108: $83-85$.

11 Tseng GF, Wang YJ, Lai QC. Perineuronal microglial reactivity following proximal and distal axotomy of rat rubrospinal neurons. Brain Research 1996; 715: $32-43$.

12 Nacimiento W et al. B-50(GAP43) in the spinal cord caudal to hemisection: indication for lack of intraspinal sprouting in dorsal root axons. Journal of Neuroscience Research 1993; 35: 603-617.

13 Liu C-N. Time pattern in retrograde degeneration after retrograde trauma of central nervous system of mammals. In: Windle WF (ed). Regeneration in the Central Nervous System. IL: Thomas, Springfield, 1954; 84-93.

14 Loewy AD, Schader RE. A quantitative study of retrograde neuronal changes in Clarke's column. Journal of Comparative Neurology 1977; 171: 65-81.

15 Bisby MA, Tetzlaff W. Changes in cytoskeletal protein synthesis following axon injury and during axon regeneration. Molecular Neurobiology 1992; 6: 107-123.

16 Barron KD. Comparative observations on the cytologic reactions of central and peripheral nerve cells to axotomy. In: Kao CC, Bunge RP, Reier PJ (eds). Spinal Cord Reconstruction. New York: Raven Press, 1983, pp. 7-39.

17 Tetzlaff W et al. Response of rubrospinal and corticospinal neurons to injury and neurotrophins. Progress in Brain Research 1994; 103: $271-286$.

18 Reid JM, Flumerfeldt BA, Gwyn DG. A cytoarchitectonic and Golgi study of the red nucleus in the rat. Journal of Comparative Neurology 1975; 162: 337-362.

19 Rodichok LD et al. Glucose utilization is unchanged in red nucleus after axotomy. Brain Research 1984; 324: 253-259.

20 Barron KD et al. Quantitative cytochemistry of RNA in axotomized feline rubral neurons. Brain Research 1977; 130: $469-481$.

21 Tetzlaff et al. Response of facial and rubrospinal neurons to axotomy: changes in mRNA expression for cytoskeletal proteins and GAP-43. Journal of Neuroscience 1991; 11: 2528-2544.

22 Jenkins R, Tetzlaff W, Hunt SP. Differential expression of immediate early genes in rubrospinal neurons following axotomy in rat. European Journal of Neuroscience 1993; 5: $203-209$.

23 Theriault E, Tetzlaff W, Tator $\mathrm{CH}$. Elevated gene expression in the red nucelus after spinal cord compression injury. Neuroreport 1992; 3: $559-562$. 
$24 \mathrm{Xu} \mathrm{XM}$, Martin GF. The response of rubrospinal neurons to axotomy in the adult opossum, Didelphis virginiana. Experimental Neurology 1990; 108: $46-54$.

25 Richardson PM, Issa VMK, Aguayo AJ. Regeneration of long spinal axons in rat. Journal of Neurocytology 1984; 13: $165-$ 182.

26 Tseng G-F, Shu J, Huang S-J, Wang Y-J. A time-dependent loss of retrograde transport ability in distally axotomized rubrospinal neurons. Anatomy and Embryology 1995; 191: 243 - 249.

27 Hung MY, Hung TJ, Shen CL. Rubrospinal tract in the Formosan monkey: HRP and autoradiographic studies. Proceedings of the National Science Council of the Republic of China. B. 1994; 18: $161-169$

28 Tseng GF, Hu ME. Axotomy induces retraction of the dendritic arbor of the adult rat rubrospinal neurons. Acta Anatomica 1996; 155: $184-193$.

29 Gwyn DG. Acetylcholinesterase activity in the red nucleus of the rat. Effects of rubrospinal tractotomy. Brain Research 1971; 35: $447-461$.
30 Gold BG, Austin DR. Regulation of aberrant neurofilament phosphorylation in neuronal perikarya. 1. Production following colchicine application to the sciatic nerve. Journal of Neuropathology and Experimental Neurology 1991; 50: 615-626.

31 Gold BG, Austin DR, Griffin JW. Regulation of aberrant neurofilament phosphorylation in neuronal perikarya. 2 . Correlation with continued axonal elongation following axotomy. Journal of Neuropathology and Experimental Neurology 1991; 50: $627-648$.

32 Pestronk A, Watson DF, Yuan CM. Neurofilament phosphorylation in peripheral nerve: Changes with axonal length and growth state. Journal of Neurochemistry 1990; 54: 977-982.

33 Tetzlaff W, Bisby MA, Kreutzberg GW. Changes in cytoskeletal proteins in the rat facial nucleus following axotomy. Journal of Neuroscience 1988; 8: $3181-3189$.

34 Tetzlaff W, Tsui BJ, Balfour JK. Rubrospinal neurons increase GAP-43 and tubulin mRNA after cervical but not after thoracic axotomy. Society of Neuroscience Abstracts 1990; 16: 338. 